\title{
Distortion Metrics of Composite Channels with Receiver Side Information
}

\author{
Yifan Liang*, Andrea Goldsmith* and Michelle Effros ${ }^{\dagger}$ \\ * Department of Electrical Engineering, Stanford University, Stanford, CA 94305 \\ $\dagger$ Department of Electrical Engineering, California Institute of Technology, Pasadena, CA 91125 \\ Email: *\{yfl, andrea\}@wsl.stanford.edu, ${ }^{\dagger}$ effros@caltech.edu
}

\begin{abstract}
We consider transmission of stationary ergodic sources over non-ergodic composite channels with channel state information at the receiver (CSIR). Previously we introduced alternative capacity definitions to Shannon capacity, including outage and expected capacity. These generalized definitions relax the constraint of Shannon capacity that all transmitted information must be decoded at the receiver. In this work alternative endto-end distortion metrics such as outage and expected distortion are introduced to relax the constraint that a single distortion level has to be maintained for all channel states. Through the example of transmission of a Gaussian source over a slow-fading Gaussian channel, we illustrate that the end-to-end distortion metrics dictate whether the source and channel coding can be separated for a communication system. We also show that the source and channel need to exchange information through an appropriate interface to facilitate separate encoding and decoding.
\end{abstract}

\section{INTRODUCTION}

End-to-end distortion is a well-accepted metric for transmission of a stationary ergodic source over stationary ergodic channels. In this work we consider transmission of a stationary ergodic source over non-ergodic composite channels. A composite channel is a collection of channels $\left\{W_{S}: S \in\right.$ $\mathcal{S}$ \} parameterized by $S$, where the random variable $S$ is chosen according to some distribution $p(S)$ at the beginning of transmission and then held fixed. We assume the channel realization is revealed to the receiver but not the transmitter. This class of channel is also referred to as the mixed channel [1] or the averaged channel [2] in literature.

The capacity of a composite channel is given by the VerdúHan generalized capacity formula [3] as $C=\sup _{\boldsymbol{X}} \underline{\boldsymbol{I}}(\boldsymbol{X} ; \boldsymbol{Y})$, where $\underline{\boldsymbol{I}}(\boldsymbol{X} ; \boldsymbol{Y})$ is the liminf in probability of the normalized information densities. This formula highlights the pessimistic nature of the Shannon capacity definition - the capacity and consequently the end-to-end distortion are dominated by the performance of the "worst" channel, no matter how small its probability. To provide more flexibility in capacity definitions, in [4], [5] we relax the constraint that all transmitted information has to be correctly decoded and derive alternative definitions including the outage and expected capacity. Previously examined in [6], outage capacity is a common criterion used in wireless fading channels. In [7] Shamai et al. also derived the expected capacity for a Gaussian slow-fading channel.

Similarly, in considering end-to-end distortion we can relax the constraint that a single distortion level has to be maintained for all channel states and introduce generalized end-to-end distortion metrics including the outage distortion and the expected distortion. The outage distortion is characterized by a pair $\left(q, D_{q}\right)$, where the distortion level $D_{q}$ is guaranteed with probability no less than $(1-q)$. This definition requires CSIR such that an outage can be declared. The expected distortion is defined as $\mathbb{E}_{S} D_{S}$, i.e. the achievable distortion $D_{S}$ in channel state $S$ averaged over the underlying distribution $p(S)$. These alternative distortion metrics are also considered in prior works. In [8] the overall distortion $q \sigma^{2}+(1-q) D_{q}$, obtained by averaging over non-outage and outage states, was adopted to analyze a two-hop fading channel. Here $\sigma^{2}$ is the variance of the source symbols. The expected distortion was also analyzed in [9]-[13] under various transmission schemes.

For transmission of a stationary ergodic source over a stationary ergodic channel, the separation theorem [14, Theorem 2.4] asserts that a target distortion level $D$ is achievable if and only if the channel capacity $C$ exceeds the source rate distortion function $R(D)$, and a two-stage separate sourcechannel code suffices to meet the requirement. However, there are examples in multi-user channels [15] where the separation theorem fails. In this work we study the separability of sourcechannel coding for generalized channel models and distortion metrics in point-to-point communications.

Source-channel separation can be defined in terms of code design. For transmission of a source over a channel the system consists of three concatenated blocks: the encoder $f_{n}$ that maps the source symbols $V^{n}$ to the channel input $X^{n}$; the channel $W^{n}$ that maps the channel input $X^{n}$ to channel output $Y^{n}$, and the decoder $\phi_{n}$ that maps the channel output $Y^{n}$ to a reconstruction of source symbols $\hat{V}^{n}$. Source-channel separation dictates that the encoder $f_{n}$ is separated into a source encoder

$$
\hat{f}_{n}: V^{n} \rightarrow\left\{1,2, \cdots, M_{s}\right\}
$$

and a channel encoder

$$
\tilde{f}_{n}:\left\{1,2, \cdots, M_{c}\right\} \rightarrow X^{n},
$$

where $M_{s} \leq M_{c}$. Similarly the decoder $\phi_{n}$ is separated into a channel decoder $\tilde{\phi}_{n}$ and a source decoder $\hat{\phi}_{n}$. In contrast joint source-channel coding is a loose label that encompasses all coding techniques where the source and channel coders are not entirely separated. Consider as an example the direct transmission of a complex circularly symmetric Gaussian source, which we denote by $\mathcal{C N}\left(0, \sigma^{2}\right)$, over a Gaussian channel with 
input power constraint $P$. The linear encoder $X=f(V)=$ $\sqrt{P / \sigma^{2}} V$ cannot be separated into a source encoder and a channel encoder. Therefore this direct transmission is an example of joint-source channel coding.

Source-channel separation implies that the operation of source and channel coding does not depend on the statistics of the counterpart. However, the source and channel do need to communicate through an interface. In the classical example of stationary ergodic sources and channels, the source requires a rate $R(D)$ based on the target distortion $D$ and the channel decides if it can support the rate based on its capacity $C$. For generalized source/channel models and distortion metrics, the interface is not necessarily a single rate and may allow multiple parameters to be agreed on between the source and channel. In [16] Vembu et al. studied the transmission of nonstationary sources over non-stationary channels. It is observed that the appropriate interface requires the notion of domination [16, Theorem 7]. Whether a source is transmissible over the channel cannot be determined by simply comparing the minimum source coding rate and channel capacity.

In this work we consider the transmission of a Gaussian source over a slow-fading Gaussian channel and illustrate that the end-to-end distortion metrics dictate whether the source and channel coding can be separated for a communication system: separation holds under the outage distortion metric but fails under the expected distortion metric. We also show that the source and channel need to exchange information through an appropriate interface, which may not be a single rate, in order to facilitate separate source-channel coding.

The rest of the paper is organized as follows. We review alternative channel capacity definitions in Section II and define generalized end-to-end distortion metrics in Section III. In Section IV we study the transmission of a Gaussian source over a slow-fading Gaussian channel. We show that the endto-end distortion metric dictates the separability of source and channel coding and also the appropriate source-channel interface. Conclusions are given in Section V.

\section{BACKGRound: Channel CAPACITy Metrics}

We review alternative channel capacity definitions derived in [4], [5] to provide some background information. In a composite channel with CSIR, the state information at the receiver can be represented as an additional output. The conditional distribution from input to output is

$$
P_{S, Y^{n} \mid X^{n}}\left(s, y^{n} \mid x^{n}\right)=P_{S}(s) P_{Y^{n} \mid X^{n}, S}\left(y^{n} \mid x^{n}, s\right) .
$$

The information density is defined similarly as in [3]

$$
i\left(x^{n} ; y^{n} \mid s\right)=\log \frac{P_{Y^{n} \mid X^{n}, S}\left(y^{n} \mid x^{n}, s\right)}{P_{Y^{n} \mid S}\left(y^{n} \mid s\right)} .
$$

\section{A. Outage Capacity}

Consider a sequence of $\left(n, 2^{n R}\right)$ codes. Let $P_{o}^{(n)}$ be the probability that the decoder declares an outage. Let $P_{e}^{(n)}$ be the probability that the receiver decodes improperly given that no outage is declared. We say that a rate $R$ is outage- $q$ achievable if there exists a sequence of $\left(n, 2^{n R}\right)$ channel codes such that $\lim _{n \rightarrow \infty} P_{o}^{(n)} \leq q$ and $\lim _{n \rightarrow \infty} P_{e}^{(n)}=0$. The capacity versus $\underset{n \rightarrow \infty}{\stackrel{n}{\text { outage }} C_{q}}$ of the above channel is defined to be the supremum over all outage- $q$ achievable rates, and is shown to be [3], [4]

$$
C_{q}=\sup _{\boldsymbol{X}} \sup \left\{\alpha: \lim _{n \rightarrow \infty} \operatorname{Pr}\left[\frac{1}{n} i\left(X^{n} ; Y^{n} \mid S\right) \leq \alpha\right] \leq q\right\} \text {. }
$$

The concept of capacity versus outage was initially proposed in [6] for cellular mobile radios. See also [17, Ch. 4] and references therein for more details. A closely-related concept of $\epsilon$-capacity was defined in [3], where the error probability $\epsilon$ consists of decoding errors unknown to the receiver. By contrast in the definition of capacity versus outage the receiver declares an outage based on CSIR when it cannot decode with vanishing error probability. As a consequence no decoding is performed for outage states.

The operational implication of this definition is that the encoder uses a single codebook and sends information at rate $C_{q}$. Assuming the channel is used repeatedly and at each use the channel state takes on some value according to $P(S)$, the receiver can correctly decode the information proportion $(1-q)$ of the time and declare an outage proportion $q$ of the time. When an outage occurs, the transmitted data are lost and the receiver may notify the sender for retransmission. We further define the outage capacity $C_{q}^{o}=(1-q) C_{q}$ as the long-term average rate, which is obtained if there is some retransmission mechanism or we consider only the fraction of correctly received packets. The value $q$ can be chosen to maximize the long-term average throughput $C_{q}^{o}$.

\section{B. Expected Capacity}

Another strategy for increasing reliably-received rate is to use a single encoder at a rate $R_{t}$ and a collection of decoders, each parameterized by $s$ and decoding at a rate $R_{s} \leq R_{t}$. The transmitter is forced to use a single encoder without channel side information, nevertheless the receiver can choose the appropriate decoder based on CSIR. Denote by $P_{e}^{(n, s)}$ the probability of error associated with channel $s$. We define the expected capacity $C^{e}$ as the supremum of all achievable rates $\mathbb{E}_{S} R_{S}$ of any code sequence that satisfies $\mathbb{E}_{S} P_{e}^{(n, S)} \rightarrow 0$.

The expected capacity of the composite channel in (1) is closely related to the capacity region of a broadcast (BC) channel with $|\mathcal{S}|$ receivers, where we denote by $|\mathcal{S}|$ the cardinality of the user index set $\mathcal{S}$. In the broadcast system the channel from the input to the output of receiver $s$ is

$$
P_{Y_{s}^{n} \mid X^{n}}\left(y_{s}^{n} \mid x^{n}\right)=P_{Y^{n} \mid X^{n}, S}\left(y_{s}^{n} \mid x^{n}, s\right) .
$$

It is easily seen that any weighted sum-rate over the broadcast capacity region is an achievable expected rate for the corresponding composite channel, where the rate $R_{s}$ achieved by user $s$ is weighted by the probability $P(s)$. Using broadcast channel codes, the expected capacity is derived in [7] for a Gaussian slow-fading channel and in [5] for a composite binary symmetric channel. General upper and lower bounds of expected capacity are also presented in [4], [5]. 


\section{End-TO-End Distortion Metrics}

We consider an stationary ergodic source that produces source symbols $V_{1}, V_{2}, \cdots, V_{n}$ drawn i.i.d. from a distribution $P(V)$. The source is transmitted over a composite channel $W^{n}: X^{n} \rightarrow\left(Y^{n}, S\right)$ with conditional output distribution

$$
W^{n}\left(y^{n}, s \mid x^{n}\right)=P_{S}(s) P_{Y^{n} \mid X^{n}, S}\left(y^{n} \mid x^{n}, s\right) .
$$

Note that the source and channel encoders, whether joint or separate, do not have access to channel state information $S$.

\section{A. Outage Distortion}

The objective is to achieve a distortion $D_{q}$ with outage probability $q$. More specifically, we want to design an encoder $f_{n}: V^{n} \rightarrow X^{n}$ that maps the source symbols to the channel input and a decoder $\phi_{n}:\left(Y^{n}, S\right) \rightarrow \hat{V}^{n}$ that maps the channel output to an estimation of source symbols such that

$$
\operatorname{Pr}\left\{\left(V^{n}, \hat{V}^{n}\right): d\left(V^{n}, \hat{V}^{n}\right) \leq D_{q}\right\} \geq 1-q,
$$

where $d\left(V^{n}, \hat{V}^{n}\right)=\frac{1}{n} \sum_{i=1}^{n} d\left(V_{i}, \hat{V}_{i}\right)$ is the distortion measure between the source sequence $V^{n}$ and its reconstruction $\hat{V}^{n}$. In order to evaluate (4) we need the conditional distribution $P\left(\hat{V}^{n} \mid V^{n}\right)$. Assuming the encoder $f_{n}$ and the decoder $\phi_{n}$ are deterministic, this distribution is given by

$\sum_{\left(X^{n}, Y^{n}, S\right)} W^{n}\left(Y^{n}, S \mid X^{n}\right) \cdot \mathbf{1}\left\{X^{n}=f_{n}\left(V^{n}\right), \hat{V}^{n}=\phi_{n}\left(Y^{n}, S\right)\right\}$

Here $\mathbf{1}\{\cdot\}$ is the indicator function. Note that the channel statistics $W^{n}$ and the source statistics $P\left(V^{n}\right)$ are fixed, so the code design is essentially the appropriate choice of the encoder-decoder pair $\left(f_{n}, \phi_{n}\right)$.

\section{B. Expected Distortion}

For the expected distortion metric, our design objective now changes from (4) to

$$
\mathbb{E}_{\left(V^{n}, \hat{V}^{n}\right)}\left\{d\left(V^{n}, \hat{V}^{n}\right)\right\} \leq D^{e},
$$

where $D^{e}$ is the target expected distortion. Using the conditional distribution $P\left(\hat{V}^{n} \mid V^{n}\right)$ in (5), the expected distortion can be rewritten as

$$
\mathbb{E}_{\left(V^{n}, \hat{V}^{n}\right)}\left\{d\left(V^{n}, \hat{V}^{n}\right)\right\}=\mathbb{E}_{S} D_{S}=\sum_{S} P(S) D_{S} .
$$

Here we denote by $D_{S}$ the achievable average distortion when the channel is in state $S$, and it is given by

$$
D_{S}=\sum P\left(V^{n}\right) W^{n}\left(Y^{n} \mid X^{n}, S\right) d\left(V^{n}, \hat{V}^{n}\right),
$$

where the summation is over all $\left(V^{n}, X^{n}, Y^{n}, \hat{V}^{n}\right)$ such that $X^{n}=f_{n}\left(V^{n}\right)$ and $\hat{V}^{n}=\phi_{n}\left(Y^{n}, S\right)$.

Notice that when a stationary ergodic source is transmitted over a stationary ergodic channel, we can design sourcechannel codes such that $d\left(V^{n}, \hat{V}^{n}\right)$ approaches the same limit as $n \rightarrow \infty$. However, in the case of a composite channel it is possible that $d\left(V^{n}, \hat{V}^{n}\right)$ approaches different limits depending on the channel state $S$, so the expected distortion metric captures the distortion averaged over various channel states.

\section{Source-Channel Coding}

In this section we consider transmission of a stationary ergodic source over non-ergodic composite channels. We first recall the definition of a source rate-distortion function as $[18$, page 342]

$$
R(D)=\min _{P(\hat{V} \mid V): \mathbb{E} d(V, \hat{V}) \leq D} I(V ; \hat{V}) .
$$

For a stationary ergodic source and channel, it is shown that if $R(D)<C$ then the source can be transmitted over the channel subject to an average fidelity criterion $\mathbb{E}\left\{d\left(V^{n}, \hat{V}^{n}\right)\right\} \leq D$. Conversely, if the transmission satisfies the average fidelity criterion, we also conclude $R(D) \leq C$ [14, page 130]. Next we consider composite channel models and generalized distortion metrics.

\section{A. Source Channel Coding under an Outage Distortion Metric}

Lemma IV.1 The source can be transmitted over the channel and satisfy the outage distortion constraint (4) if

$$
R\left(D_{q}\right)<C_{q}=C_{q}^{o} /(1-q),
$$

where $C_{q}^{o}$ is the outage capacity, $C_{q}$ is defined in (3) and $R\left(D_{q}\right)$ is the source rate distortion function (7) evaluated at distortion level $D_{q}$.

This lemma gives a sufficient condition for the source to be transmitted over the channel subject to the outage distortion constraint (4). In the proof we see the design of encoder $f_{n}$ involves a two-stage procedure, i.e. a source encoder $\hat{f}_{n}$ and a channel encoder $\tilde{f}_{n}$, and similarly for the decoder $\phi_{n}$. In fact Lemma IV.1 can be viewed as the direct part of source-channel separation under the outage distortion metric.

In the rate distortion theory for source coding, one often imposes the average fidelity criterion

$$
\mathbb{E}\left\{d\left(V^{n}, \hat{V}^{n}\right)\right\} \leq D .
$$

The main challenge here is to satisfy the condition (4) which is based on the tail of the distortion distribution rather than on its mean. So for source coding, instead of the global average fidelity criterion (8), we impose the following local $\epsilon$-fidelity criterion [14, page 123]

$$
\operatorname{Pr}\left\{\left(V^{n}, \hat{V}^{n}\right): d\left(V^{n}, \hat{V}^{n}\right) \leq D\right\} \geq 1-\epsilon .
$$

It is well known that for any $\delta>0$ there exist source codes with rate $R<R(D)+\delta$ such that the average fidelity criterion is satisfied [18, page 351]. In order to prove Lemma IV.1, we need the following stronger result [14, page 125]:

Lemma IV.2 For any $0<\epsilon<1$ there exist source codes with rate $R<R(D)+\delta$ that satisfy the $\epsilon$-fidelity criterion (9).

The existence of these codes is essential to the following proof of Lemma IV.1.

Proof: In the following we denote $R=R\left(D_{q}\right)$ and $C=C_{q}=C_{q}^{o} /(1-q)$ to simplify notation. By Lemma IV.2, for any $0<\epsilon<1$ and $\delta>0$, there exists source encoder

$$
\hat{f}_{n}: V^{n} \rightarrow U \in\left\{1,2, \cdots, 2^{n(R+\delta)}\right\}
$$


and source decoder

$$
\hat{\phi}_{n}: U \in\left\{1,2, \cdots, 2^{n(R+\delta)}\right\} \rightarrow \tilde{V}^{n}
$$

such that $\operatorname{Pr}\left\{d\left(V^{n}, \tilde{V}^{n}\right) \leq D\right\} \geq 1-\epsilon$. Here $\tilde{V}^{n}$ is the source reconstruction sequence. By definition of $C=C_{q}$ there exist channel codes with channel encoder

$$
\tilde{f}_{n}: U \in\left\{1,2, \cdots, 2^{n(C-\delta)}\right\} \rightarrow X^{n}
$$

and channel decoder

$$
\tilde{\phi}_{n}:\left(Y^{n}, S\right) \rightarrow \hat{U} \in\left\{1,2, \cdots, 2^{n(C-\delta)}\right\}
$$

such that $\lim _{n \rightarrow \infty} P_{o}^{(n)} \leq q$ and $\lim _{n \rightarrow \infty} P_{e}^{(n)}=0$. For sufficiently small $\delta$ we have $R+\delta<C-\delta \vec{~}$, which guarantees the output of the source encoder $\hat{f}_{n}$ always lies in the domain of the channel encoder $\tilde{f}_{n}$.

Now we concatenate the source encoder, channel encoder, channel decoder and source decoder to form a communication system. Denote by $V^{n}$ and $\hat{V}^{n}$ the original and reconstructed source sequences, respectively. We have

$$
\begin{aligned}
& \operatorname{Pr}\left\{d\left(V^{n}, \hat{V}^{n}\right) \leq D\right\} \\
\geq & \operatorname{Pr}\left\{d\left(V^{n}, \hat{V}^{n}\right) \leq D, U=\hat{U}\right\} \\
= & \operatorname{Pr}\{U=\hat{U}\} \cdot \operatorname{Pr}\left\{d\left(V^{n}, \tilde{V}^{n}\right) \leq D\right\} \\
\geq & \left(1-P_{o}^{(n)}\right)\left(1-P_{e}^{(n)}\right)(1-\epsilon) \rightarrow 1-q
\end{aligned}
$$

as $n \rightarrow \infty$ and $\epsilon \rightarrow 0$.

Note that although Lemma IV.1 and IV.2 are derived for sources with finite alphabets and bounded distortion measures, the result presented here can be generalized to continuousalphabet sources and unbounded distortion measures using the technique of [19, Ch. 7].

For our strategy the outage states are recognized by the receiver, which can request a retransmission or simply reconstruct the source symbol by its mean - hence the distortion is the variance of the source symbol. The same outage distortion constraint (4) can be also met by concatenating the source code in Lemma IV.2 and the channel code based on the $\epsilon$-capacity of [3]. However, there is a subtle difference: the receiver cannot recognize the decoding error in the latter strategy and the reconstruction based on the decoded symbols, possibly in error, may lead to large distortions.

We illustrate the separate source and channel codes constructed in the proof of Lemma IV.1 by the following example. As shown in Figure 1, a Gaussian source $\mathcal{C N}\left(0, \sigma^{2}\right)$ is transmitted over a Rayleigh slow-fading Gaussian channel with fading distribution $p(\gamma)=(1 / \bar{\gamma}) e^{-\gamma / \bar{\gamma}}$, where $\bar{\gamma}$ is the average channel power gain. The transmitter has a power constraint $P$. The additive Gaussian noise is i.i.d. and normalized to have unit variance. In this example we index each channel by the power gain $\gamma$, which has the same role as the previous channel index $s$. We consider the case where the source block length is the same as the channel block length, i.e. the bandwidth expansion ratio $b$, defined as the number of channel uses per source symbol, equals to 1 .
For an outage probability $q$ the corresponding threshold of channel gain is $\gamma_{q}=-\bar{\gamma} \log (1-q)$, so in non-outage states the channel can support a rate of

$$
C_{q}=\log \left(1+P \gamma_{q}\right)=\log [1-P \bar{\gamma} \log (1-q)]
$$

The rate distortion function of a complex Gaussian source is given by $R\left(D_{q}\right)=\log \left(\sigma^{2} / D_{q}\right)$. From Lemma IV.1 if

$$
\sigma^{2} / D_{q}<1-P \bar{\gamma} \log (1-q),
$$

then the outage distortion requirement (4) can be satisfied by concatenation of a source code at rate $R\left(D_{q}\right)$ and a channel code at rate $C_{q}$ as given in (10).

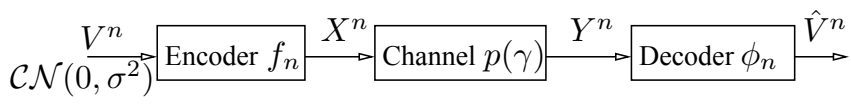

Fig. 1. Transmission of Gaussian source over slow-fading Gaussian channels

It is well known that the uncoded scheme is optimal for transmission of a Gaussian source over a Gaussian channel when the bandwidth expansion ratio $b=1$ [11], [20]. The optimality is in the sense that a linear code $X=\sqrt{P / \sigma^{2}} V$ can achieve the minimum distortion

$$
D_{\gamma}^{*}=\frac{\sigma^{2}}{1+P \gamma}
$$

for each channel state $\gamma$. It is easily seen that the optimal uncoded scheme also requires (11) in order to satisfy the outage distortion constraint.

For the system under consideration, we have shown that separate source-channel coding meets the distortion constraint (4) if $R\left(D_{q}\right)<C_{q}$; if $R\left(D_{q}\right)>C_{q}$ then the outage distortion constraint can never be met even for joint source-channel coding. The result can be extended to slow-fading Gaussian channels with any fading distribution $p(\gamma)$, not necessarily the Rayleigh fading.

For other systems that transmit stationary ergodic sources over composite channels, Lemma IV.1 gives the direct part of the source-channel separation under the outage distortion metric. In order to prove optimality of separate designs we need to show that the outage distortion criterion cannot be met even with joint source-channel coding if $R\left(D_{q}\right)>C_{q}$. This converse is work in progress.

\section{B. Source-Channel Separation Fails for Expected Distortion}

Unlike the outage distortion metric, we do not believe that source-channel separation holds for the expected distortion metric. The same example in Figure 1 can be used to illustrate this. In the following we give the achievable expected distortion with optimal uncoded transmissions and also analyze the distortion under separate source-channel coding.

We have assumed a bandwidth expansion ratio $b=1$ in the example. Note that even the simplest problem of transmitting a Gaussian source over a two-user degraded Gaussian broadcast channel under bandwidth compression or expansion $(b \neq 1)$ is still open. Many schemes based on layering and hybrid analogdigital transmission have been proposed to tackle the problem 
[9]-[11], but so far no generally optimal scheme or general converse to the distortion region is known.

1) Optimal Joint Source-Channel Coding: As aforementioned in Section IV-A, the uncoded scheme with a linear code $X=\sqrt{P / \sigma^{2}} V$ can achieve the minimum distortion (12) for each channel state $\gamma$, and therefore achieves the optimal expected distortion

$$
\left(D^{e}\right)^{*}=\int_{0}^{\infty} \frac{\sigma^{2} e^{-\gamma / \bar{\gamma}}}{1+P \gamma} \cdot \frac{d \gamma}{\bar{\gamma}}=\frac{\sigma^{2} e^{1 / P \bar{\gamma}}}{P \bar{\gamma}} \operatorname{Ei}\left(\frac{1}{P \bar{\gamma}}\right),
$$

with $\operatorname{Ei}(x)=\int_{x}^{\infty}\left(\frac{e^{-t}}{t}\right) d t$ the exponential integral function.

2) Source-Channel Separation with Channel Codes for Outage Capacity: Consider using a channel code for outage capacity $C_{q}^{o}$ and a source code at rate $C_{q}=C_{q}^{o} /(1-q)$ with $C_{q}$ defined in (3). With probability $q$ the channel is in outage so the receiver estimates the transmitted source symbol by its mean and the distortion is its variance $\sigma^{2}$. With probability $(1-q)$ the channel can support the rate $C_{q}$ and the end-to-end distortion is $D_{q}=D\left(C_{q}\right)$. The overall expected distortion is averaged over the non-outage and outage states, i.e. $D_{1}^{e}(q)=q \sigma^{2}+(1-q) D_{q}$.

Under separate source-channel coding and channel codes for outage capacity, the minimum achievable distortion is obtained by optimizing $D_{1}^{e}(q)$ over $q \in(0,1)$. For the example in Figure 1 this becomes

$$
D_{1}^{e}=\min _{0<q<1} D_{1}^{e}(q)=\min _{0<q<1} q \sigma^{2}+\frac{(1-q) \sigma^{2}}{1-P \bar{\gamma} \log (1-q)}
$$

and the solution is to evaluate $D_{1}^{e}(q)$ at

$$
q_{D}^{*}=1-\exp \left\{-\frac{2}{1+\sqrt{1+4 P \bar{\gamma}}}\right\} .
$$

One might be tempted to think that the channel should optimize its outage capacity

$$
\max _{0<q<1} C_{q}^{o}=\max _{0<q<1}(1-q) \log [1-P \bar{\gamma} \log (1-q)]
$$

and provide $\left(q_{C}^{*}, R_{q_{C}^{*}}\right)$ as the interface to the source, where $q_{C}^{*}$ is the argument that maximizes (16). In fact the solution

$$
q_{C}^{*}=1-\exp \left\{-\frac{e^{W(P \bar{\gamma})}-1}{P \bar{\gamma}}\right\},
$$

with $W(z)$ the Lambert- $W$ function solving $z=W(z) e^{W(z)}$, is in general different from $q_{D}^{*}$ in (15). It is insufficient for the channel to provide only $\left(q_{C}^{*}, R_{q_{C}^{*}}\right)$ as the interface; instead it should provide the entire $\left(q, C_{q}\right)$ curve and let the source choose the optimal operating point on this curve to minimize overall average distortion. Similarly, given a target expected distortion $D$, the source should determine for each outage probability $q$ the corresponding outage distortion $D_{q}=$ $\left(D-q \sigma^{2}\right) /(1-q)$ and provide the entire $\left(q, R\left(D_{q}\right)\right)$ curve as the interface. With separate source-channel coding and a channel code for outage capacity, the expected distortion target is achievable if and only if $R\left(D_{q}\right) \leq C_{q}$ for some $q$.

We illustrate the source-channel interface with a numerical example of the communication system in Figure $1: \bar{\gamma}=1$, $\sigma^{2}=1$ and $P=10$. From (14) the minimum expected distortion $D_{1}^{e}=0.443$ is obtained with $q_{D}^{*}=0.237$. For three different expected distortion levels $D=\left\{0.9 D_{1}^{e}, D_{1}^{e}, 1.1 D_{1}^{e}\right\}$, we compute for each outage probability $q$ the corresponding outage distortion $D_{q}=\left(D-q \sigma^{2}\right) /(1-q)$ and the source coding rate $R\left(D_{q}\right)$. These curves are plotted in Figure 2 together with the outage capacity $C_{q}^{o}$ and the rate $C_{q}$ that can be supported by the channel for non-outage states. We observe the outage capacity is maximized at $q_{C}^{*}=0.38 \neq q_{D}^{*}$, so in general we should compare the entire curve $\left(q, C_{q}\right)$ and $\left(q, R\left(D_{q}\right)\right)$ to determine whether the expected distortion target can be achieved with channel codes for outage capacity.

3) Source-Channel Separation with Channel Codes for Expected Capacity: We have seen in Section II that a composite channel can be viewed as a broadcast channel with virtual receivers indexed by each channel state. A broadcast channel code can be applied to achieve rate $R_{s}$ when channel is in state $s$. It is well-known that a Gaussian source is successively refinable so we can design a multi-resolution source code which, when combined with the broadcast channel code, achieves a distortion $D\left(R_{s}\right)$ for each channel state $s$. The overall expected distortion is $\mathbb{E}_{S} D\left(R_{S}\right)$.

For the system under consideration, we assume a power allocation $\rho(\gamma) \geq 0$ which satisfies the overall power constraint $\int_{0}^{\infty} \rho(\gamma) d \gamma=P$. It is shown in [7] that the following rate is achievable

$$
R(\gamma)=\int_{0}^{\gamma} \frac{u \rho(u)}{1+u I(u)} d u
$$

when the channel gain is $\gamma$. Here $I(\gamma)=\int_{\gamma}^{\infty} \rho(u) d u$ is the interference level when channel is in state $\gamma$. The minimum expected distortion with a multi-resolution source code and a broadcast channel code is then

$$
\min _{\rho(\gamma)} \int_{0}^{\infty} \sigma^{2} e^{-R(\gamma)} p(\gamma) d \gamma
$$

The optimization problem (17) was solved in [13]. The optimal power allocation satisfies

$$
\rho_{D}^{*}(\gamma)= \begin{cases}0, & \gamma<\gamma_{P} \text { or } \gamma>\bar{\gamma} \\ -I^{\prime}(\gamma), & \gamma_{P} \leq \gamma \leq \bar{\gamma}\end{cases}
$$

where

$$
I(\gamma)=\frac{\int_{\bar{\gamma}}^{\gamma}\left(\frac{1}{2 \bar{\gamma}}-\frac{1}{u}\right) e^{-u / 2 \bar{\gamma}} d u}{\gamma e^{-\gamma / 2 \bar{\gamma}}}
$$

and $\gamma_{P}$ satisfies $I\left(\gamma_{P}\right)=P$. The minimum expected distortion is

$$
D_{2}^{e}=\sigma^{2}\left[D\left(\gamma_{P}\right)+\int_{0}^{\gamma_{P}} p(\gamma) d \gamma\right]
$$

where

$$
D(\gamma)=\frac{e^{-1}-\frac{1}{\bar{\gamma}} \int_{\bar{\gamma}}^{\gamma} e^{-(u+\bar{\gamma}) / 2 \bar{\gamma}}(u / \bar{\gamma})^{-1} d u}{(\gamma / \bar{\gamma})^{-1} e^{(\gamma-\bar{\gamma}) / 2 \bar{\gamma}}}
$$

In general the optimal power allocation $\rho_{C}^{*}(\gamma)$ that maximizes the expected capacity $\int_{0}^{\infty} R(\gamma) p(\gamma) d \gamma$, as determined in [7], is different from $\rho_{D}^{*}(\gamma)$ that minimizes the expected distortion (17). Assuming separate source and channel coding and a 
broadcast channel code, the channel should provide the entire capacity region $\left\{\left(R_{s}\right)\right\}$ as the interface.

In Figure 3 we plot the expected distortion under the different source-channel coding schemes explored in this section. It is observed that the broadcast channel code combined with the multi-resolution source code performs slightly better than the channel code for outage capacity combined with a single rate source code, but there is a large gap between their expected distortion and that of the optimal uncoded scheme.

It is known that source-channel separation fails for certain multi-user channels [15]. Here we consider transmission of a Gaussian source over a slow-fading Gaussian channel and illustrate that even for point-to-point communication systems, under certain end-to-end distortion metrics such as expected distortion, separation also fails and joint source-channel coding is necessary to achieve the optimal performance.

\section{CONCLUSION}

We consider transmission of a stationary ergodic source over non-ergodic composite channels with channel state information at the receiver (CSIR). Similar to previously studied alternative channel capacity definitions such as outage and expected capacity, alternative end-to-end distortion metrics including outage and expected distortion are introduced in this work. We then study the transmission of a Gaussian source over slow-fading Gaussian channels and illustrate that the source-channel coding can be separated under an end-to-end outage distortion metric, while joint source-channel coding is optimal under an expected distortion metric. We also show that the source and channel need to exchange information through an appropriate interface in order to facilitate separate sourcechannel coding.

\section{REFERENCES}

[1] T. S. Han. Information-Spectrum Method in Information Theory. Applications of mathematics. Springer, New York, NY, 2003.

[2] R. Ahlswede. The weak capacity of averaged channels. Z. Wahrscheinlichkeitstheorie und Verw. Gebiete, 11:61-73, 1968.

[3] S. Verdú and T. Han. A general formula for channel capacity. IEEE Trans. Inform. Theory, 40(4):1147-1157, July 1994.

[4] M. Effros and A. Goldsmith. Capacity definitions and coding strategies for general channels with receiver side information. In IEEE ISIT, page 39, Cambridge MA, August 1998.

[5] M. Effros, A. Goldsmith and Y. Liang. Capacity definitions of general channels with receiver side information. In IEEE ISIT, Nice, France, June 2007.

[6] L. Ozarow, S. Shamai and A. Wyner. Information theoretical considerations for cellular mobile radio. IEEE Trans. Veh. Tech., 43(2):359-378, May 1994.

[7] S. Shamai and A. Steiner. A broadcast approach for a single-user slowly fading MIMO channel. IEEE Trans. Inform. Theory, 49(10):2617-2635, Oct. 2003.

[8] K. Zachariadis, M. Honig and A. Katsaggelos. Source fidelity over a two-hop fading channel. In IEEE MilCom, pages 134-139, Monterey CA, Nov. 2004.

[9] S. Shamai, S. Verdú and R. Zamir. Systematic lossy source/channel coding. IEEE Trans. Inform. Theory, 44(2):564-579, March 1998

[10] Z. Reznic, M. Feder and R. Zamir. Distortion bounds for broadcasting with bandwidth expansion. IEEE Trans. Inform. Theory, 52(8):37783788, August 2006.

[11] U. Mittal and N. Phamdo. Hybrid digital-analog (HDA) joint sourcechannel codes for broadcasting and robust communications. IEEE Trans. Inform. Thoery, 48(5):1082-1102, May 2002.
[12] D. Gündüz and E. Erkip. Joint source channel codes for MIMO block fading channels. Submitted to IEEE. Trans. Inform. Theory, March 2006.

[13] C. Ng, D. Gündüz, A. Goldsmith and E. Erkip. Minimum expected distortion in Gaussian layered broadcast coding with successive refinement. In IEEE ISIT, Nice, France, June 2007. To appear.

[14] I. Csiszár and J. Körner. Information Theory: Coding Theorems for Discrete Memoryless Systems. Academic Press, New York, 1981.

[15] T. Cover, A. El Gamal and M. Salehi. Muliple access channels with arbitrarily correlated sources. IEEE Trans. Inform. Theory, 26(6):648657, Nov. 1980

[16] S. Vembu, S. Verdú and Y. Steinberg. The source-channel separation theorem revisited. IEEE Trans. Inform. Theory, 41(1):44-54, Jan. 1995.

[17] A. Goldsmith. Wireless Communications. Cambridge University Press, New York NY, 2005.

[18] T. Cover. Broadcast channels. IEEE Trans. Inform. Theory, 18:2-14, Jan. 1972.

[19] R. Gallager. Information Theory and Reliable Communication. New York: Wiley, 1968.

[20] M. Gastpar, B. Rimoldi and M. Vetterli. To code, or not to code: lossy source-channel communication revisited. IEEE Trans. Inform. Theory, 49(5):1147-1158, May 2003.

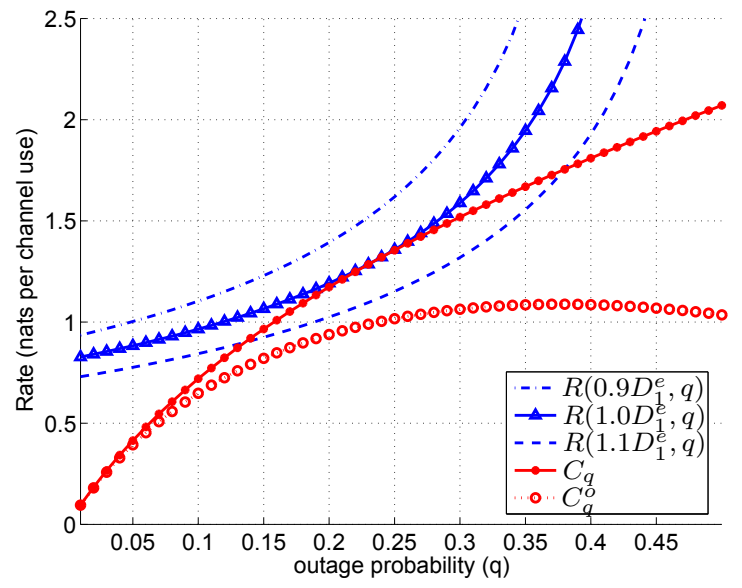

Fig. 2. Source channel interface with channel code for outage capacity

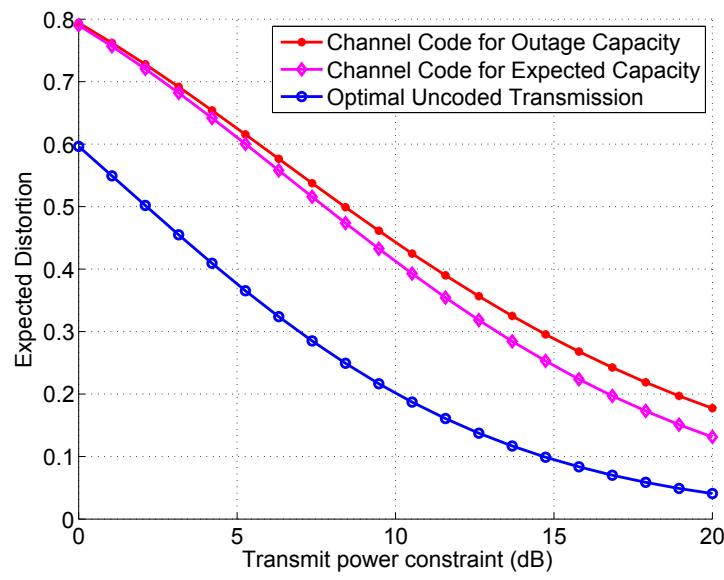

Fig. 3. Expected distortion for various source-channel coding schemes 